\title{
EURE 1970-2008: breve genealogía de su trayectoria*
}

\author{
FELIPE LINK L. ** \\ **Sociólogo. Magíster en Investigación Social y Desarrollo. Candidato a Doctor en Arquitectura y Estudios \\ Urbanos, Pontificia Universidad Católica de Chile.
}

\section{Introducción}

Al cumplirse casi cuarenta años del primer número publicado de la revista EURE y en pleno proceso de modernización e incorporación de nuevas tecnologías para la presentación y difusión de sus contenidos, se me encargó el trabajo de revisión y asignación de palabras descriptoras a cada uno de los más de 500 trabajos publicados entre 1970 y 2008. La idea inicial era generar los insumos necesarios para la construcción de un índice temático de la revista, con el objetivo de maximizar el rendimiento de búsquedas, así como mejorar los índices de impacto y difusión de cada artículo.

Esta inmensa tarea analítica y clasificatoria arrojó una serie de resultados bastante sorprendentes, que superaron con creces el objetivo inicial de los productos encomendados, e hicieron también mucho más interesante el trabajo, que por momentos se asemejó a una arqueología de la propia revista, desentrańando ideas, relaciones y conceptos articulados en diferentes trabajos intermitentes en el tiempo.

Los resultados inesperados están siempre presentes en cualquier trabajo de investigación, muchas veces constituyen caminos desconocidos e inciertos, pero que dan razón de ser a la estructura rigurosa y detallada de la planificación inicial, sin la cual serían imposibles dichos hallazgos. En palabras de Merton (1980), los resultados no buscados en un proceso de investigación, distan mucho de ser resultados indeseables y muchas veces constituyen el cuerpo central de las teorías de alcance medio. Estos hallazgos repentinos, frutos de una "casualidad planificada" y conocidos por su expresión en inglés como serendipity, constituyen una excelente forma de entender la evolución y persistencia de EURE desde 1970 hasta hoy.

Así, en este proceso largo y a veces pesado de revisión de textos y asignación de descriptores, nos encontramos con bastantes resultados esperados, entre otros, la primacía de algunos conceptos centrales y aglutinadores de los contenidos en cada artículo, según las diferentes coyunturas sociopolíticas en el continente. Dentro de estos conceptos, podemos señalar: planificación del desarrollo, desarrollo regional y local, globalización, medioambiente y sustentabilidad, etc. Así también, aparecen otros hallazgos más extraños, inesperados, pero no indeseables siguiendo a Merton, como son algunos artículos referidos a regiones y países más lejanos o poco conocidos

* Correspondencia: Felipe Link, El Comendador 1970, Providencia, Santiago, Chile. E-mail: falink@uc.cl 
en el contexto de los estudios urbanos y regionales latinoamericanos, como Sri Lanka, Corea, Jamaica o Japón, que quizás hoy día serían publicados en otras revistas especializadas.

Con todo, el objetivo de este breve trabajo es mostrar el proceso de autoobservación de la revista en los 102 números publicados, así como describir los temas más recurrentes, datos estadísticos y otros hallazgos peculiares entre la gran diversidad de artículos y autores que han formado parte de EURE a lo largo de su historia.

\section{Temas y contenidos}

El procedimiento de asignación de descriptores se planificó como un proceso absolutamente inductivo, es decir, de análisis de los textos y registro de los términos más adecuados a cada temática particular. En este sentido, se dejó de lado la referencia a la asignación propia de palabras clave de cada autor, que por lo demás, existe sistemáticamente sólo desde el número 74 de la revista, esto es, desde 1999, así como también se privilegió el análisis del texto propiamente tal, por sobre el resumen y el título. De esta manera, se logró identificar 12 grandes áreas temáticas relacionadas a EURE, que agrupan más de 300 descriptores particulares. Estos grandes temas son: i) Economía urbana y regional; ii) Desarrollo regional y local; iii) Desarrollo urbano y territorial; iv) Política urbana y regional; v) Planificación y teoría urbana; vi) Estructura urbana y organización espacial; vii) Vivienda social y segregación; viii) Transporte urbano; ix) Pobreza y conflicto social; x) Medioambiente y sustentabilidad; xi) Globalización; xii) Historia y cultura urbana.

Dada la forma como se llegó a esta clasificación, es probable que no sea la definitiva para efectos editoriales ${ }^{1}$. Sin embargo, es bastante útil al momento de describir la evolución general de la revista. Como se puede observar en el siguiente gráfico, las áreas de interés más recurrentes son: economía urbana y regional, desarrollo regional y local, y desarrollo urbano y territorial, sin perjuicio de que cada artículo puede inscribirse en más de un área temática.

1 En efecto, el trabajo realizado se encuentra actualmente en proceso de arbitraje por expertos. 
Figura 1. Frecuencia de áreas temáticas

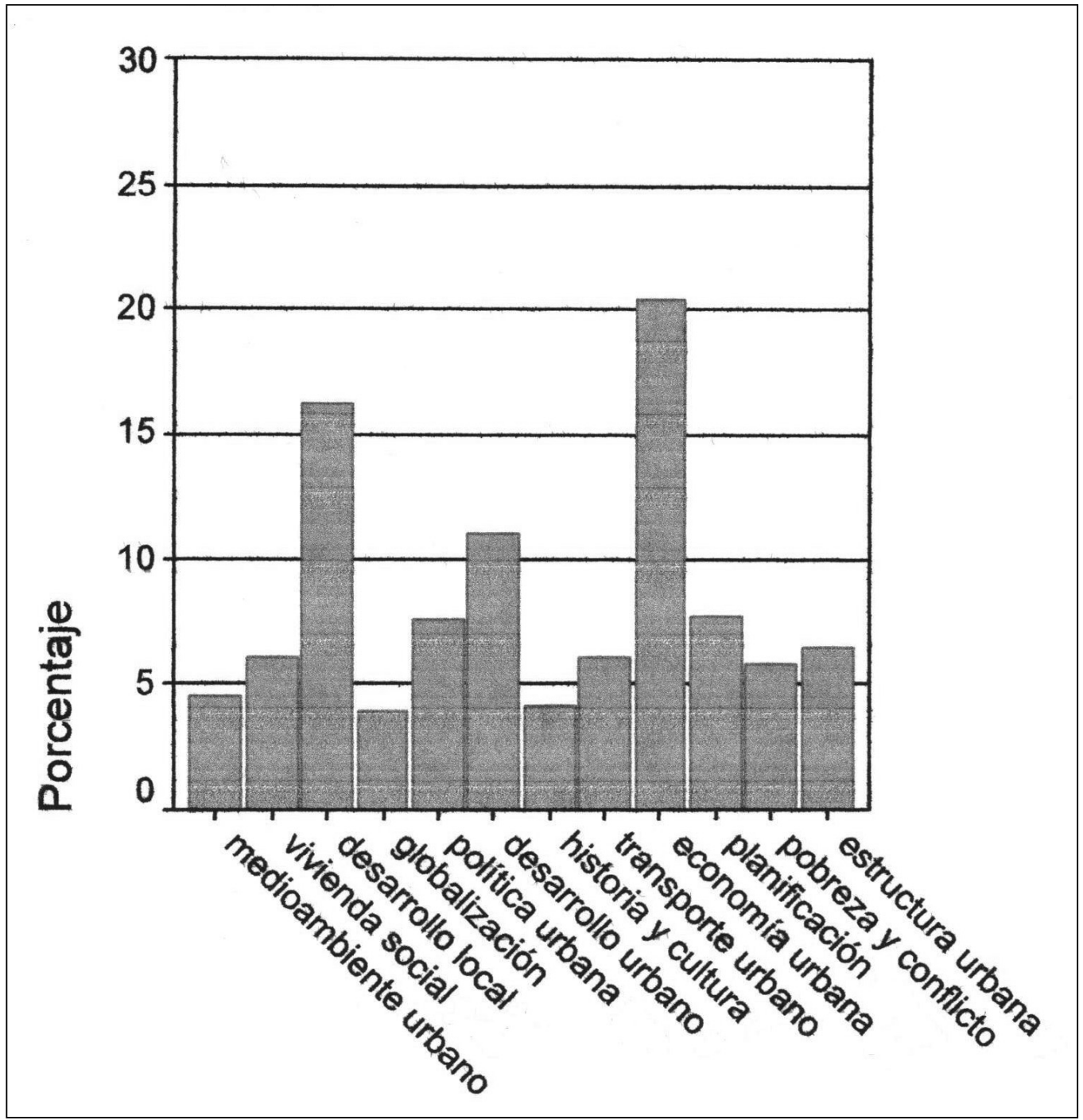

Fuente: Elaboración propia

A pesar de la predominancia general de algunos temas, existen diferencias importantes según el año de publicación de la revista. Así, es esperable la emergencia en las últimas décadas de trabajos relacionados con sustentabilidad o con los procesos de globalización. También, fueron más recurrentes los artículos dedicados a la planificación en la década de 1970 y al desarrollo en la de 1980. La siguiente tabla muestra esta evolución de los contenidos de EURE. 
Cuadro 1. Evolución de áreas temáticas

\begin{tabular}{|c|c|c|c|c|c|c|c|}
\hline & \multicolumn{4}{|c|}{ década de la revista } & \multirow[b]{2}{*}{ Total } \\
\hline & & & $\begin{array}{l}\text { década } \\
\text { de } 1970\end{array}$ & $\begin{array}{l}\text { década } \\
\text { de } 1980\end{array}$ & $\begin{array}{l}\text { década } \\
\text { de } 1990\end{array}$ & $\begin{array}{l}\text { década } \\
\text { de } 2000\end{array}$ & \\
\hline \multirow{24}{*}{$\begin{array}{l}\text { tema } \\
\text { descriptor }\end{array}$} & \multirow[t]{2}{*}{ medioambiente urbano } & Recuento & 1 & 7 & 7 & 9 & 24 \\
\hline & & $\%$ de tema descriptor & $4,2 \%$ & $29,2 \%$ & $29,2 \%$ & $37,5 \%$ & $100,0 \%$ \\
\hline & \multirow{2}{*}{$\begin{array}{l}\text { vivienda social y } \\
\text { segregación }\end{array}$} & Recuento & 4 & 8 & 6 & 14 & 32 \\
\hline & & $\%$ de tema descriptor & $12,5 \%$ & $25,0 \%$ & $18,8 \%$ & $43,8 \%$ & $100,0 \%$ \\
\hline & \multirow[t]{2}{*}{ desarrollo local y regional } & Recuento & 10 & 31 & 28 & 17 & 86 \\
\hline & & $\%$ de tema descriptor & $11,6 \%$ & $36,0 \%$ & $32,6 \%$ & $19,8 \%$ & $100,0 \%$ \\
\hline & \multirow[t]{2}{*}{ globalización } & Recuento & 0 & 0 & 6 & 15 & 21 \\
\hline & & $\%$ de tema descriptor &, $0 \%$ &, $0 \%$ & $28,6 \%$ & $71,4 \%$ & $100,0 \%$ \\
\hline & \multirow[t]{2}{*}{ política urbana y regional } & Recuento & 5 & 15 & 10 & 10 & 40 \\
\hline & & $\%$ de tema descriptor & $12,5 \%$ & $37,5 \%$ & $25,0 \%$ & $25,0 \%$ & $100,0 \%$ \\
\hline & \multirow{2}{*}{$\begin{array}{l}\text { desarrollo urbano y } \\
\text { territorial }\end{array}$} & Recuento & 13 & 15 & 16 & 14 & 58 \\
\hline & & $\%$ de tema descriptor & $22,4 \%$ & $25.9 \%$ & $27,6 \%$ & $24,1 \%$ & $100,0 \%$ \\
\hline & \multirow{2}{*}{$\begin{array}{l}\text { historia y cultura urbana y } \\
\text { regional }\end{array}$} & Recuento & 2 & 3 & 6 & 11 & 22 \\
\hline & & $\%$ de tema descriptor & $9,1 \%$ & $13,6 \%$ & $27,3 \%$ & $50,0 \%$ & $100,0 \%$ \\
\hline & \multirow[t]{2}{*}{ transporte urbano } & Recuento & 5 & 9 & 10 & 8 & 32 \\
\hline & & $\%$ de tema descriptor & $15,6 \%$ & $28,1 \%$ & $31,3 \%$ & $25,0 \%$ & $100,0 \%$ \\
\hline & \multirow{2}{*}{$\begin{array}{l}\text { economia urbana y } \\
\text { regional }\end{array}$} & Recuento & 15 & 21 & 39 & 33 & 108 \\
\hline & & $\%$ de tema descriptor & $13,9 \%$ & $19,4 \%$ & $36,1 \%$ & $30,6 \%$ & $100,0 \%$ \\
\hline & \multirow{2}{*}{$\begin{array}{l}\text { planificación y teoría } \\
\text { urbana y regional }\end{array}$} & Recuento & 16 & 8 & 12 & 5 & 41 \\
\hline & & $\%$ de tema descriptor & $39,0 \%$ & $19,5 \%$ & $29,3 \%$ & $12,2 \%$ & $100,0 \%$ \\
\hline & \multirow[t]{2}{*}{ pobreza y conflicto social } & Recuento & 8 & 10 & 7 & 6 & 31 \\
\hline & & $\%$ de tema descriptor & $25,8 \%$ & $32,3 \%$ & $22,6 \%$ & $19,4 \%$ & $100,0 \%$ \\
\hline & \multirow{2}{*}{$\begin{array}{l}\text { estructura urbana y } \\
\text { organización espacial }\end{array}$} & Recuento & 9 & 6 & 10 & 9 & 34 \\
\hline & & $\%$ de tema descriptor & $26,5 \%$ & $17,6 \%$ & $29,4 \%$ & $26,5 \%$ & $100,0 \%$ \\
\hline \multirow[t]{2}{*}{ Total } & & Recuento & 88 & 133 & 157 & 151 & 529 \\
\hline & & $\%$ de tema descriptor & $16.6 \%$ & $25,1 \%$ & $29,7 \%$ & $28,5 \%$ & $100,0 \%$ \\
\hline
\end{tabular}

Fuente: Elaboración propia

Es interesante constatar la incorporación creciente de trabajos relacionados con historia y cultura urbana y regional, así como la disminución de artículos sobre pobreza urbana y conflicto social. Si bien, la mayoría de los trabajos sobre vivienda social y segregación, que aumentan considerablemente en el tiempo, efectivamente abordan la pobreza y el conflicto social, lo hacen desde una perspectiva menos crítica que en décadas pasadas. Por último, cabe destacar la persistencia de ciertos temas, aunque con enfoques diferentes, según la contingencia. Por ejemplo, los artículos clasificados en política urbana y regional, se refieren, en los últimos años, a la discusión operativa sobre políticas públicas, mientras que en el pasado, desarrollaban una discusión política más conceptual.

Los trabajos relacionados con desarrollo constituyen también una parte importante de los artículos de EURE. Sin embargo, queda en evidencia la ambivalencia y multiplicidad de este concepto, ya que generalmente es necesario especificar la dimensión a la que se refiere. En este sentido, encontramos, entre otros, desarrollo: regional, urbano, rural, territorial, sustentable, económico, social, nacional, local, industrial, endógeno, etc. Por lo tanto, es un término que necesita ser precisado, ya que además, se encuentra también como teoría del desarrollo, planificación del desarrollo y finalmente desarrollo, a secas. 
Ahora, tomando como referencia esta vez los títulos de todos los artículos publicados, se logró construir un esquema de relación semántica, a partir de la cuantificación de los términos incluidos en cada título ${ }^{2}$. Este esquema, se realizó para cada década de la revista, con el fin de observar, a través de los propios títulos y no de los descriptores asignados, la recurrencia de los temas publicados.

Figura 2. Esquema conceptual en la década de 1970.

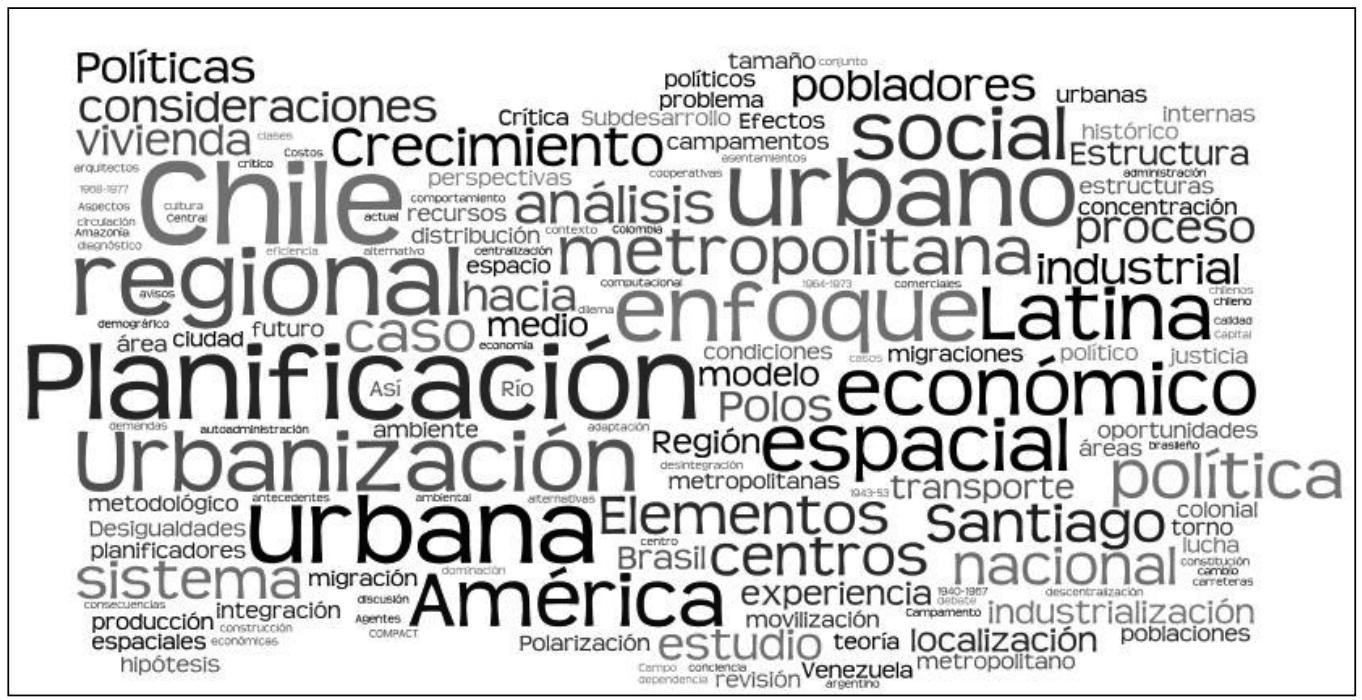

Fuente: Elaboración propia

Como se puede observar, en los setenta, los trabajos publicados se enfocan en temas relacionados con planificación, desarrollo regional, Chile y América Latina. Se observan, además, algunos términos sugerentes como: pobladores, industrial, vivienda y desigualdades, lo que evidentemente da cuenta de los problemas de investigación de la época.

\footnotetext{
Este procedimiento se efectuó a través de la herramienta wordle, disponible en http://wordle.net y excluyó la palabra desarrollo, por ser la más recurrente en todos los períodos y por lo tanto, poco discriminante. En el esquema, las palabras de mayor tamaño, corresponden a las mayores frecuencias, mientras que las de menor tamaño, a las que registran menores apariciones.
} 
Figura 3. Esquema conceptual en la década de 1980.

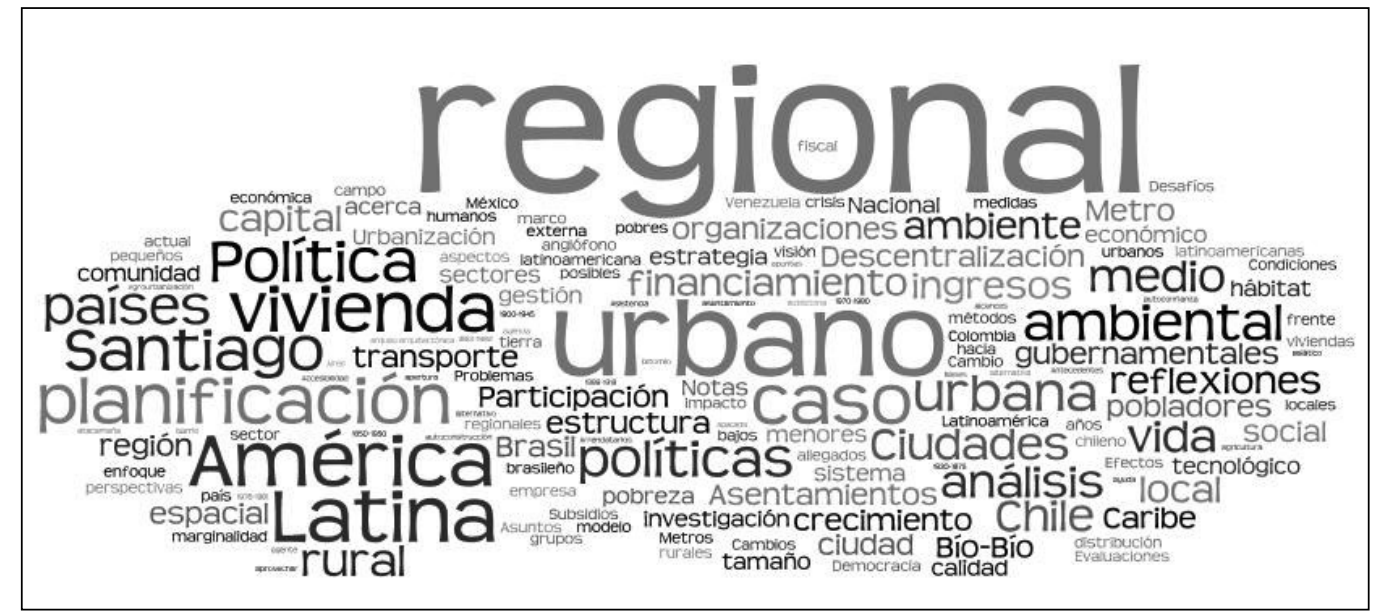

Fuente: Elaboración propia

A diferencia de la década anterior, en los ochenta, aparecen tímidamente algunas palabras como: ambiental, vida y transporte, lo que da cuenta de la emergencia de temas nuevos en la investigación urbana. Sin embargo, persisten todavía los trabajos referidos a la planificación y al análisis regional.

Figura 4. Esquema conceptual en la década de 1990.

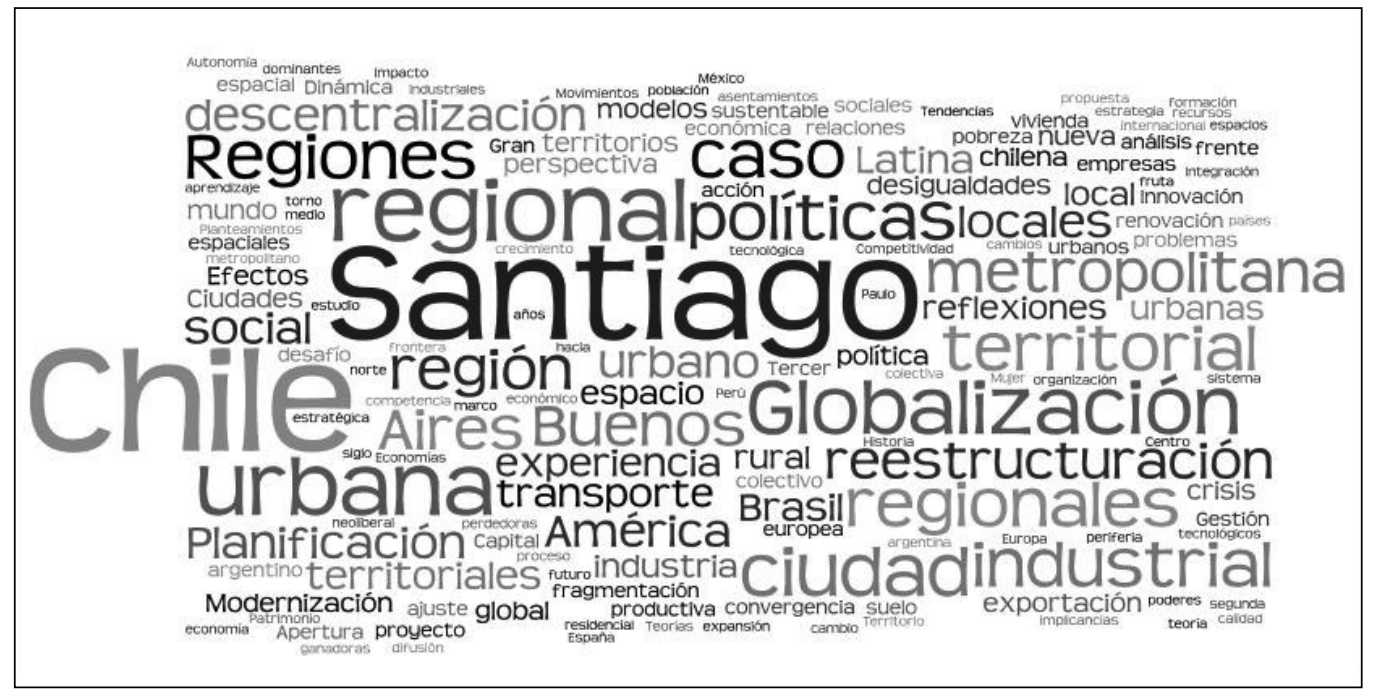

Fuente: Elaboración propia 
En los noventa, aparecen con fuerza los trabajos sobre globalización y reestructuración económica. Predominan los artículos referidos a Chile y específicamente a Santiago y se observa curiosamente la recurrencia de la palabra "caso", lo que puede indicar la emergencia de los estudios de caso para contextualizar la discusión.

Figura 5. Esquema conceptual en la década de 2000.

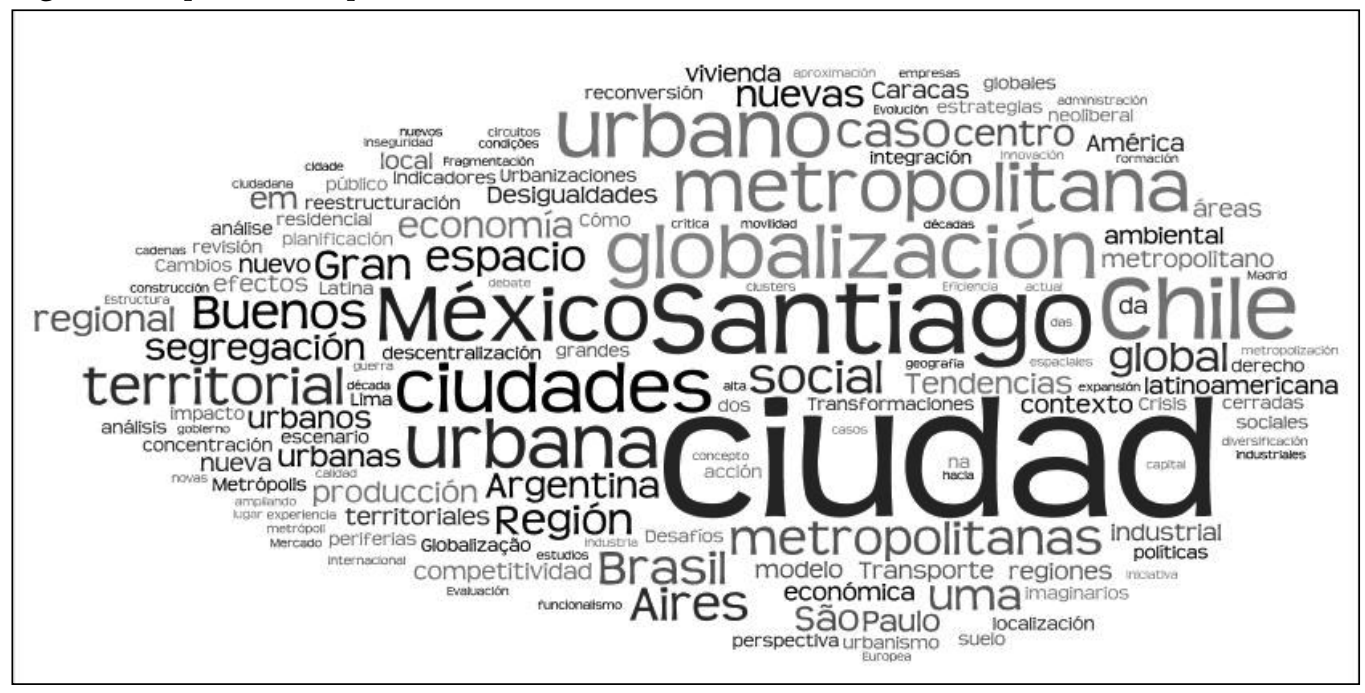

Fuente: Elaboración propia

Por último, la década actual consolida a la globalización como tema importante, además de centrar los trabajos en la ciudad y lo urbano, por sobre la predominancia regional de las décadas anteriores. También, se observa una mayor diversidad en los casos referidos, apareciendo Buenos Aires, Sao Paulo, Caracas, Brasil, México y Argentina como los más frecuentes junto a Chile y Santiago.

Es decir, sin entrar en las múltiples interpretaciones posibles de estos esquemas, vemos que la evolución temática de los trabajos publicados, interpretada a partir de los propios títulos de cada artículo, es coherente con la definición de áreas temáticas realizada a partir de la asignación de descriptores. Por lo tanto, podemos identificar el paso desde el análisis de la planificación regional y urbana, hacia los estudios sobre globalización y ciudad en sentido amplio, pasando por la discusión sobre desarrollo regional y urbano. Esto es coherente con la evolución sociopolítica de América Latina y los conflictos predominantes en cada período, lo que se traduce generalmente en problemas de investigación social. Siguiendo a Mills (1959), los problemas de investigación social, surgen de una operacionalización conceptual de los problemas sociales, aunque no se correspondan necesariamente con ellos. Es decir, que los problemas de investigación social están íntimamente ligados al contexto y a los conflictos sociales predominantes, intentando muchas veces dar cuenta de sus causas y consecuencias. 


\section{Autores y trabajos publicados}

En las cuatro décadas de la revista, se han publicado 529 artículos centrales -esto es, sin contar reseñas, tribunas o editoriales-, con un promedio de 5,4 artículos por número.

Figura 6. Artículos por década

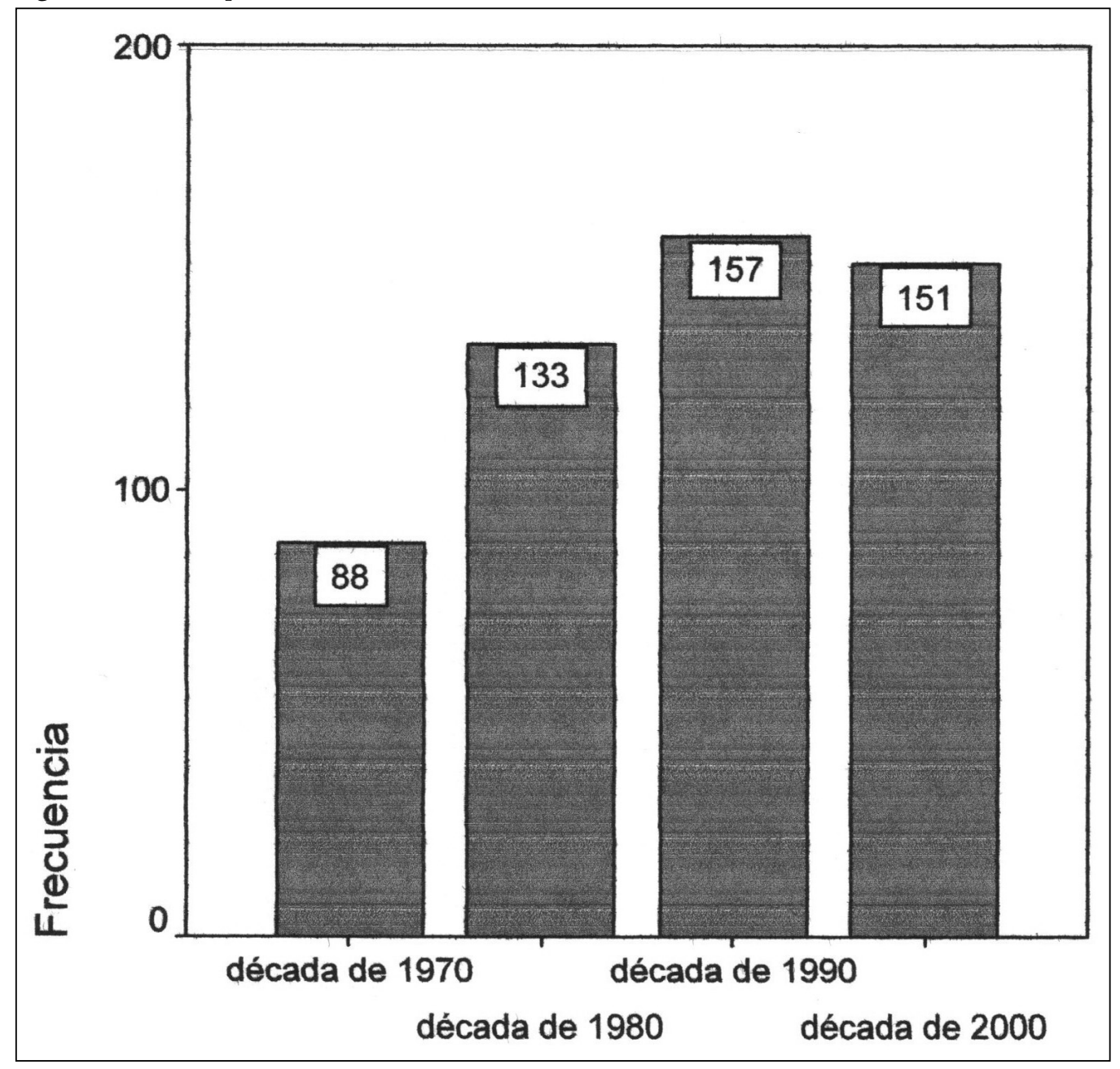

Fuente: Elaboración propia

Si bien, podemos observar una tendencia creciente en la cantidad de artículos publicados por la revista3, también es cierto que han existido momentos de escasa productividad y muchas dificultades para mantener la continuidad. Este es el caso, por ejemplo, de los primeros años de dictadura, donde se publicaron números con 2 ó 3 artículos. El siguiente gráfico muestra

3 Cabe señalar que la década de 2000 sólo considera hasta el presente año y hasta el número 102, es decir, que todavía faltan cerca de 25 trabajos por publicar hasta 2009. 
la cantidad de trabajos publicados anualmente desde 1970, donde es evidente la disminución entre los ańos 1976 y 1980.

Figura 7. Artículos por año

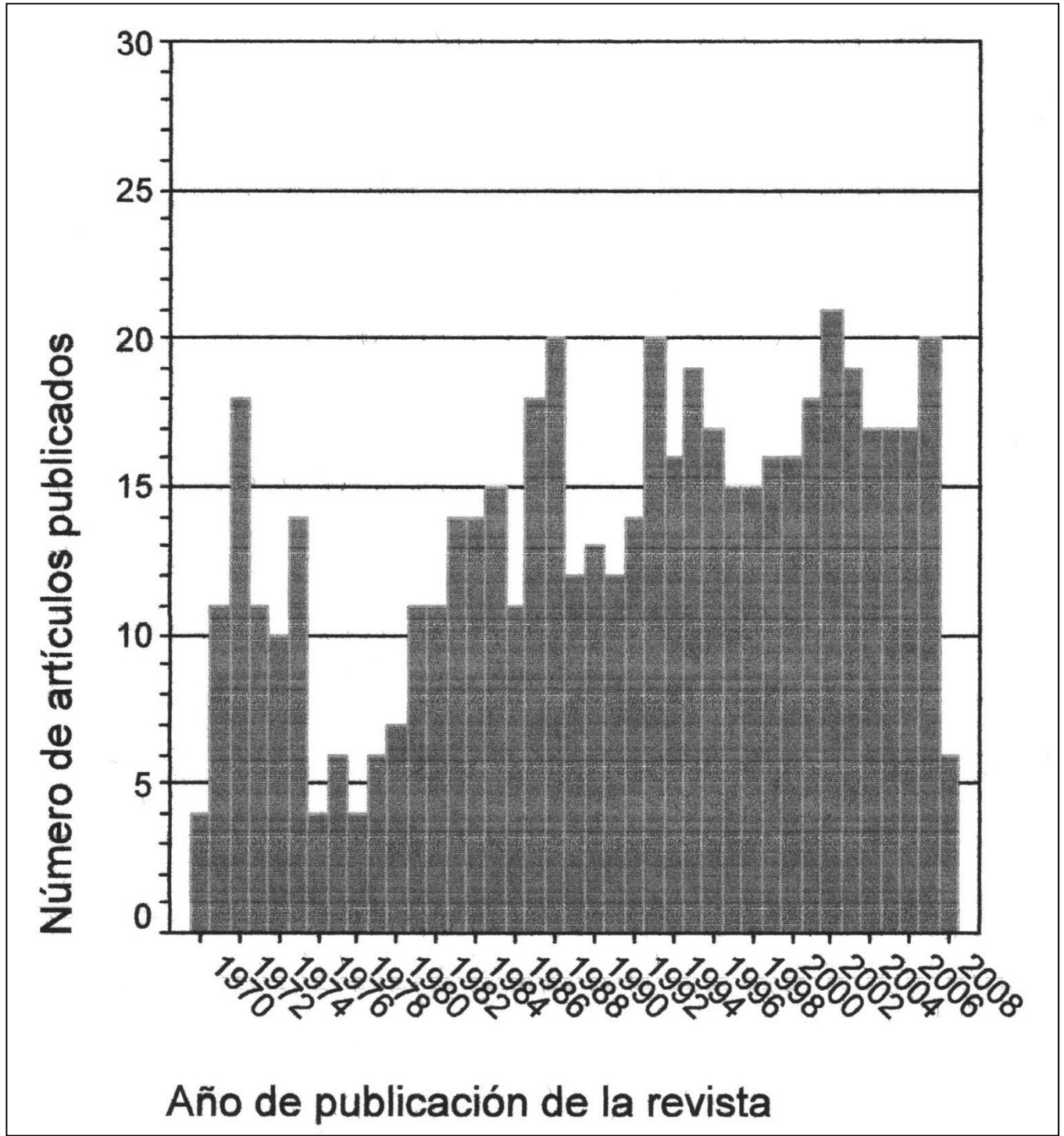

Fuente: Elaboración propia

Otros datos interesantes tienen que ver con los autores que contribuyen a la revista. Se puede observar una predominancia de trabajos individuales, lo que no contribuye a la consolidación de grupos de investigación e integración de investigadores jóvenes. Por una parte, el carácter interdisciplinario de los estudios urbanos y territoriales, hace necesario un trabajo integrado de diferentes investigadores y disciplinas, complementando datos, métodos, técnicas y teorías, en un proceso de triangulación en la investigación (Bericat, 1998), que finalmente se refleje en las publicaciones periódicas. Por otra parte, los investigadores jóvenes se potencian al ser incluidos 
en los resultados publicados de trabajos donde muchas veces actúan como colaboradores en diferentes etapas de la investigación, sea recolectando, procesando o analizando datos.

Figura 8. Autoría de los artículos

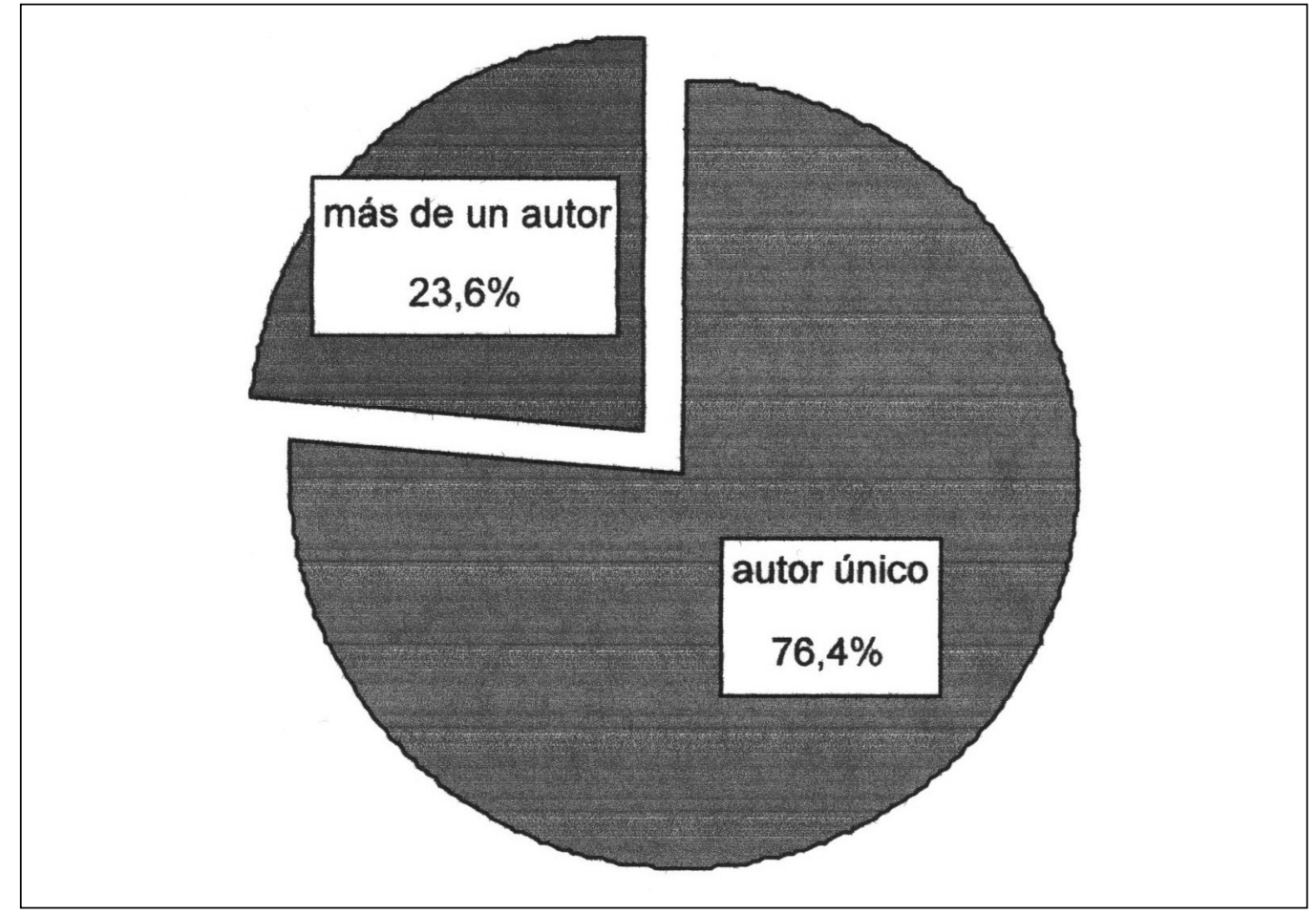

Fuente: Elaboración propia

Como se ve, sólo un 23,6\% de los trabajos son colectivos y de este porcentaje, que corresponde a 125 trabajos, sólo 43 trabajos involucran a más de dos autores ${ }^{4}$. Es decir, que hay una tendencia importante hacia el trabajo individual, sin incorporar colaboradores. A pesar de esta tendencia, existen algunas diferencias significativas entre países. Si bien no se registró la nacionalidad de los autores en la base de datos construida para este trabajo, sí se registró el país al que los artículos hacen una referencia explícita, por lo que entenderemos que los autores representan a esos países en la gran mayoría de los casos. Siendo así, vemos que los autores españoles y brasileños, han trabajado mucho más colectivamente que el resto, como se observa en el Cuadro 2.

433 trabajos involucran a tres autores, 8 trabajos a cuatro autores y sólo 2 trabajos a cinco autores. 
Cuadro 2. Autores por país



Fuente: Elaboración propia 
Cuadro 3. Autores por tema

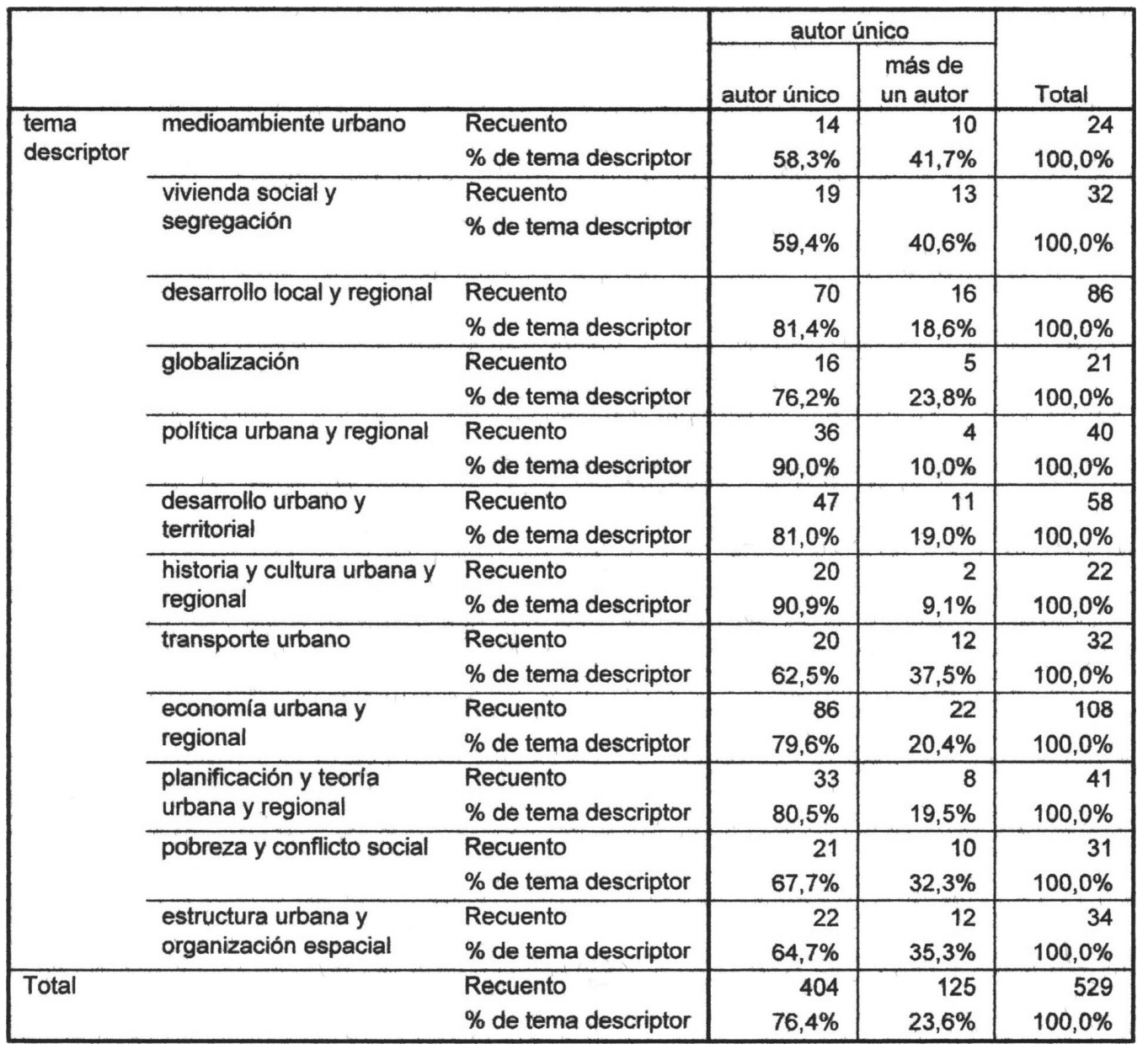

Fuente: Elaboración propia

El Cuadro No3 es sugerente también, ya que muestra la relación entre colectividad de los trabajos y temas de investigación ${ }^{5}$. Podemos observar que algunos temas como politica urbana o historia y cultura urbana y regional, son mayoritariamente individuales, mientras que medioambiente urbano y vivienda social y segregación, contribuyen con trabajos colectivos. Esto puede tener relación con la consolidación e importancia de ciertos temas de investigación, lo que lleva a constituir grupos de trabajo en los diferentes centros de investigación y universidades en nuestro continente.

\footnotetext{
5 Cabe señalar que todas las tablas presentadas son estadísticamente significativas, a través de la prueba de chi cuadrado. Es decir, no se trataría de relaciones aleatorias, sino de diferencias reales.
} 
Otro dato importante de considerar, en relación a los autores, tiene que ver con las diferencias de género. De los 529 autores principales, sólo un 15\% son mujeres, lo que aumenta a un $18 \%$ cuando se consideran todos los autores, principales y secundarios.

Figura 9. Distribución por sexo de los autores principales

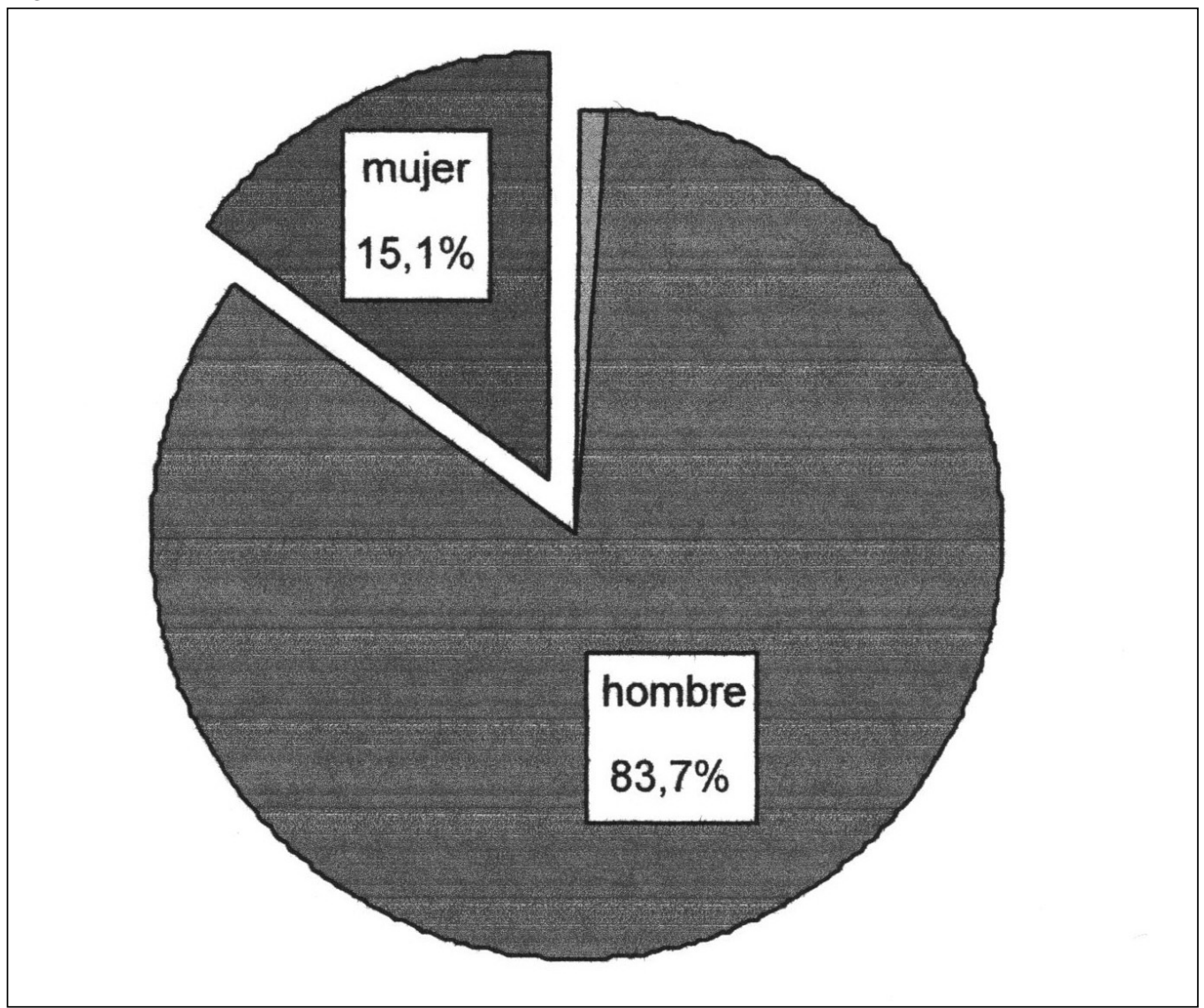

Fuente: Elaboración propia

A pesar de esta disparidad en la composición por género entre los investigadores, es notable el aumento de las autoras en los últimos años. De esta manera, si en los '70 representaban menos de un 5\%, en los últimos ańos sobrepasan el 30\% ${ }^{6}$.

$6 \quad$ El primer grupo de la tabla corresponde a trabajos donde el autor es un grupo, por lo que no es posible diferenciar por género. Por ejemplo, CEPAL, CIDU, etc. 
Cuadro 4. Sexo de los autores según década de publicación

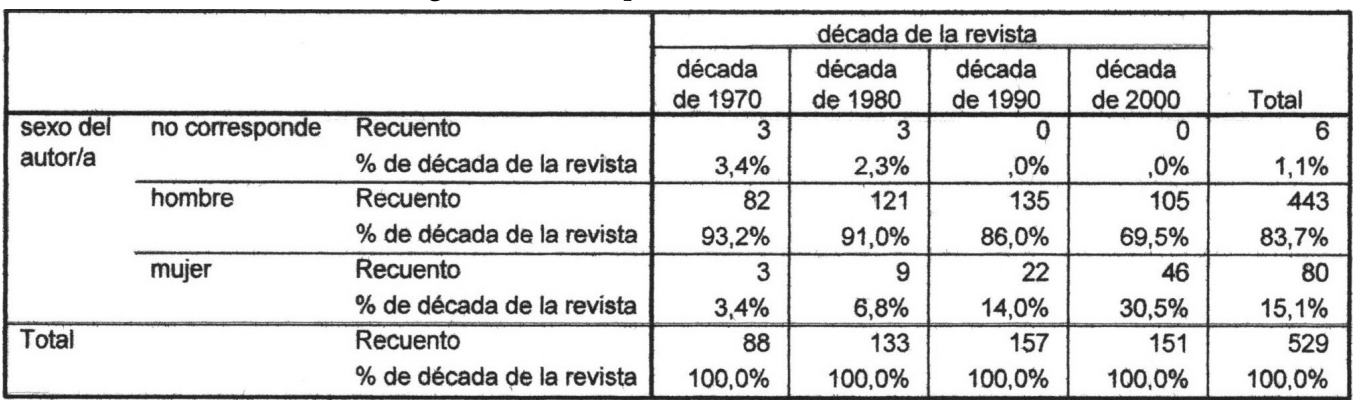

Fuente: Elaboración propia

Curiosamente, las investigadoras presentan algunas características interesantes, como por ejemplo, ser más inclusivas, con un mayor porcentaje de trabajos colectivos. Por otra parte, ellas se dedican más a algunos temas de investigación como globalización y medioambiente, mientras que prácticamente no aparecen en artículos referidos a planificación.

Cuadro 5. Trabajos colectivos según sexo

\begin{tabular}{|c|c|c|c|c|c|c|}
\hline & & & \multicolumn{3}{|c|}{ sexo del autor/a } & \multirow[b]{2}{*}{ Total } \\
\hline & & & $\begin{array}{c}\text { no } \\
\text { corresponde }\end{array}$ & hombre & mujer & \\
\hline \multirow[t]{4}{*}{ autor único } & autor único & Recuento & 6 & 343 & 55 & 404 \\
\hline & & $\%$ de sexo del autor/a & $100,0 \%$ & $77,4 \%$ & $68,8 \%$ & $76,4 \%$ \\
\hline & más de un autor & Recuento & 0 & 100 & 25 & 125 \\
\hline & & $\%$ de sexo del autor/a &, $0 \%$ & $22,6 \%$ & $31,3 \%$ & $23,6 \%$ \\
\hline \multirow{2}{*}{\multicolumn{2}{|c|}{ Total }} & Recuento & 6 & 443 & 80 & 529 \\
\hline & & $\%$ de sexo del autor/a & $100,0 \%$ & $100,0 \%$ & $100,0 \%$ & $100,0 \%$ \\
\hline
\end{tabular}

Fuente: Elaboración propia

Por último, si bien es cierto que existen bastantes autores que tienen entre cinco y más de diez artículos publicados en la revista, es claro que la gran mayoría solamente publica una vez. De hecho, es impresionante el número de 453 autores en 529 trabajos publicados.

\section{Ciudades y territorios}

Como se mencionó, existe una gran diversidad de ciudades y países referidos en los artículos de EURE. Si bien hay una cantidad importante (25\% aproximadamente) que no se refieren a ningún caso particular, es decir, que son ensayos temáticos y más generales, en el $75 \%$ restante se refleja el carácter latinoamericano de esta publicación. 
Figura 10. País o región referida en los textos

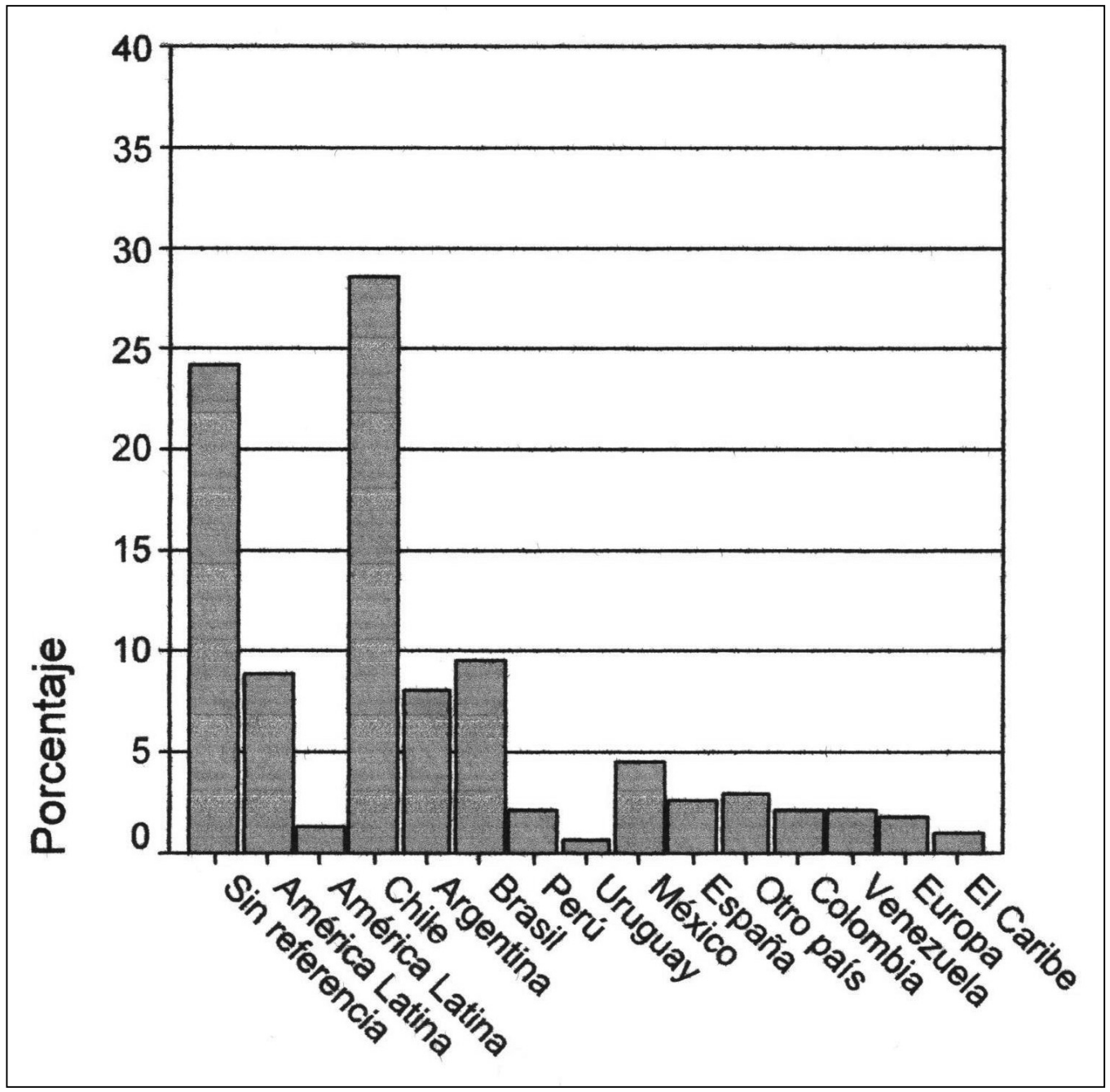

Fuente: Elaboración propia

Como se ve, la presencia de trabajos sobre América Latina, Brasil, Argentina y México es muy importante, principalmente en los últimos años. Dentro de las ciudades más recurrentes están, Santiago (70 artículos), Buenos Aires (22 artículos), Sao Paulo (11 artículos) y Ciudad de México (9 artículos). Estos corresponden a trabajos donde se considera explícitamente a dichas ciudades como el contexto en el cual se desarrolla el artículo, entendiendo que es probable que estén referidas también tangencialmente en otros trabajos. En total, se clasificaron 68 territorios con referencia explícita en los artículos, entre ciudades, regiones, países o continentes. Entre los más inesperados se encuentran África, Baltimore, Corea, Chiloé, Jamaica, Japón, Pomaire, Sri Lanka o Teherán.

Por último, el carácter territorial de los trabajos presentados en EURE es indiscutible. Si bien, la mayoría de los artículos no presenta resultados de investigaciones empíricas ni datos de 
fuentes primarias, prácticamente todos tienen al territorio, en cualquiera de sus escalas, como un factor determinante para el análisis, logrando reunir una gran cantidad de material para comprender los procesos socio-espaciales de nuestro continente.

Para concluir, basta señalar nuevamente que el objetivo de este trabajo fue describir la trayectoria de la revista EURE, a través de hallazgos colaterales a un trabajo editorial pragmático en función de objetivos muy diferentes; $y$, en este contexto, motivar la reflexión acerca de la importancia de la revista en el escenario latinoamericano y los desafíos futuros en relación con la cooperación y trabajo colectivo entre los investigadores, la difusión de investigaciones empíricas de datos primarios, la inclusión de investigadores jóvenes, la actualización de la reflexión crítica en torno a los problemas de investigación, la interdisciplinariedad de las publicaciones, entre muchos otros. Esto es, adoptar a grandes rasgos el nuevo rol de los intelectuales en una época de transición (Wallerstein, 2001).

\section{Referencias bibliográficas}

Bericat, E. (1998). La integración de los métodos cuantitativo y cualitativo en la investigación social. Barcelona: Ariel Sociología.

Merton, R. K. (1980). Ambivalencia sociológica y otros ensayos. Madrid: Editorial Espasa.

Mills, Ch. W. (1959). La imaginación sociológica. México: FCE.

Revista Latinoamericana de Estudios Urbanos Regionales (EURE) (1970-2008). Instituto de Estudios Urbanos y Territoriales, Pontificia Universidad Católica de Chile, Santiago.

Wallerstein, I. (2001). Los intelectuales en una época de transición. Cuadernos Universidad Pontificia de Salamanca, Programa Guatemala, Revista Semestral 1. 